\title{
Motivos del desuso de puentes peatonales en Arequipa
}

\section{Motives of disuse of pedestrian bridges in Arequipa}

\author{
MsC. Walter L. Arias Gallegos \\ Universidad Católica San Pablo. Arequipa, Perú.
}

\section{RESUMEN}

Objetivo: conocer las causas por las cuales las personas no utilizan los puentes peatonales de la ciudad de Arequipa.

Métodos: estudio descriptivo realizado a través de la aplicación de una encuesta que recoge datos sobre la edad, género, grado de instrucción, procedencia y experiencia de accidentes previos. Se realizó un muestreo no probabilístico mediante la técnica de muestro por cuotas. Se pesquisaron 360 peatones, 270 hombres y 90 mujeres, que transitan por las vías aledañas a tres puentes de la ciudad: 1. El puente de la Avenida Venezuela, 2. El puente de la Avenida Alcides Carrión, y 3. El puente de la Avenida Ejército.

Resultados: la edad promedio de la muestra era de 31,97 años y el grado de instrucción no fue determinante en el uso de los puentes. Se encontraron causas particulares para cada puente, lo que implica que su ubicación es relevante en la toma de decisiones de los peatones que los usan. La falta de tiempo y la pereza fueron los motivos más frecuentes que señalaron las personas para no utilizar los puentes peatonales.

Conclusiones: es importante colocar bardas que eviten transitar por la pista indebidamente y realizar campañas de concientización que brinden información a los peatones sobre los riesgos a que se enfrentan por cruzar indebidamente la calle y por infringir la ley.

Palabras clave: Psicología del tránsito, conductas de riesgo, peatón, puentes peatonales. 
ABSTRACT

Objective: to find out the causes of disuse of pedestrian bridges in Arequipa city. Methods: descriptive study based on a survey gathering data on age, gender level of education, origin and experience of previous accidents. A non-probabilistic sampling was performed through quota sampling technique. Three hundred and sixty pedestrians ( 270 males and 90 females), who moved along pathways next to three city bridges such as Avenida Venezuela bridge, Avenida Alcides Carriion bridge and Avenida Ejercito bridge, were studied.

Results: average age of the sample was 31.97 years, the level of education did not influence the use of bridges. Lack of time, sloth and the fear for attacks and robbery were the most frequent motives marked by pedestrians as causes for ignoring the pedestrian bridges.

Conclusions: it is necessary to take measures in terms of security and surveillance at bridges, as well as launching awareness-raising campaigns that provide information to pedestrians on the possible risks of wrongly crossing the streets and of breaking the law.

Key words: Traffic psychology, risky behavior, pedestrian, pedestrian bridges.

\section{NTRODUCCI ÓN}

\section{Psicología del tránsito, accidentabilidad y seguridad vial}

La seguridad vial es un concepto que cobra vital importancia en la actualidad, debido al incremento de unidades vehiculares del parque automotor por una parte, y por otra, al elevado número de accidentes de tránsito que se registran día a día en Perú y otros países. En ese sentido, usualmente se considera un sistema tripartito en el que intervienen factores mecánicos, ambientales y humanos como componentes interactuantes de la seguridad vial, pero el factor humano está presente en el $90 \%$ de los siniestros, ${ }^{1}$ lo que supone, al menos de parte de los psicólogos, poner especial énfasis en este factor.

Precisamente, aunque la psicología del tránsito participa del estudio de estos tres tipos de factores, se centra en el último -es decir, en el componente humano-. De modo que, abordando el complejo problema de la seguridad vial con una metodología rigurosa, la psicología estudia la conducta del hombre en los diversos escenarios que se relacionan con las conductas seguras e inseguras (o de riesgo), tanto de los conductores como de los peatones.

En el Perú los accidentes de tránsito alcanzan cifras alarmantes, y ello se debe a la falta de conciencia de seguridad vial, o peor aún, a la ausencia de una cultura de seguridad que se manifieste en las diversas esferas de la vida cotidiana como el hogar, el trabajo y en el tránsito vial, ya sea como peatones o como conductores. Aunque este problema no es exclusivo de Perú, y mucho menos de América Latina, es en Sudamérica que las estadísticas rebasan los registros que se tienen en todo el globo. 
Mundialmente 1,2 millones de personas mueren al año en accidentes de tránsito, ${ }^{2}$ de tal modo que en el mundo muere una persona cada 15 min debido a los accidentes de tránsito. En los Estados Unidos la cifra pasa a 94 muertes por día. Más aún, si se consideran las causas de los accidentes de tránsito, estas se deben principalmente a la imprudencia del conductor o del peatón. Así, el $25 \%$ de todos los accidentes normales, tiene que ver con una persona haciendo uso del celular, pero el mayor porcentaje se relaciona directamente con el consumo de alcohol. Concretamente, en relación con los accidentes de tráfico y de manera general, se estima que el consumo de alcohol es responsable del 30 al $50 \%$ de los accidentes con víctimas mortales, del 15 al $35 \%$ de los que causan lesiones graves, y del 10 $\%$ de los que no causan lesiones.

Todo esto supone que dichos accidentes podrían evitarse si se tuviese una conciencia de seguridad vial. La seguridad vial está orientada a la prevención de los accidentes de tránsito o la minimización de sus efectos, especialmente para la vida y la salud de las personas. En ese sentido, las normas reguladoras de tránsito y la responsabilidad de los usuarios de la vía pública componen la piedra angular de la seguridad vial. Sin una organización por parte del Estado y sin la moderación de las conductas humanas no es posible lograr óptimos resultados.

Es precisamente la prevención el factor que resulta crucial para evitar la accidentabilidad de los peatones y los conductores. Dentro de este marco de prevención, los factores culturales y educativos podrían jugar un papel preponderante, ya que los bajos niveles culturales, educacionales y de valores de la población de América Latina podrían explicar los altos niveles de accidentabilidad. Anualmente se registran 125000 muertes ocasionadas por accidentes de tránsito en América Latina, lo que representa el $4 \%$ del PBI de la región por concepto de atención a las víctimas. De modo que los accidentes de tránsito constituyen además de un problema social y de salud, un problema económico, y por supuesto educativo. De ahí que la educación vial sea imprescindible en la escuela y en el hogar.

En Perú se registran 30 muertes por cada 10000 habitantes ocasionadas por accidentes de tránsito. En México son 28, en Colombia 24, en Chile 7 y en Argentina 5; la mayoría de los muertos son peatones. El promedio de América Latina y el Caribe es de 17 muertos por accidentes de tránsito por cada 10000 habitantes, por lo que alcanza el doble del promedio de víctimas mortales que el promedio mundial.

\section{Conductas de riesgo en los puentes peatonales de Arequipa}

Una de las principales causas de accidentes de tránsito recae sobre las conductas imprudentes de los peatones. Dentro del marco de investigación de la psicología del tránsito, la conducta de los peatones y sus actitudes son fundamentales, ${ }^{3}$ aunque también se deben considerar diversos aspectos como el diseño urbano y la normativa legal que regula y sanciona la conducta de los peatones. Precisamente, para contrarrestar la alta incidencia de conductas de riesgo del peatón, el 15 de diciembre de 2010 entró en vigencia en el Perú, el Decreto Supremo DS 040-2010 que modifica el reglamento de tránsito (Ley № 23463) para multar a los peatones imprudentes.

Sin embargo, el diseño urbano es importante, más aún en ciudades como Arequipa donde las calzadas son estrechas. En ese sentido, en esta ciudad se han venido haciendo diversas reformas y modificaciones urbanas caracterizadas por la mejora 
estructural de calles y veredas, así como la peatonalización de diversas calles del centro histórico de la ciudad. Rivas, ${ }^{4}$ refiere que para 70000 peatones que transitan por la ciudad, se requieren $50000 \mathrm{~m}^{2}$ de vereda, pero en Arequipa (en el perímetro que abarca la zona de las calles Pizarro, Cruz verde, Ayacucho y Alto de la luna) solo hay $30000 \mathrm{~m}^{2}$ de vereda. Esto explica por qué 6 de cada 10 peatones caminan por la calle.

Por otro lado, los conductores no suelen respetar al peatón. De hecho, los estudios de Conejera y otros, ${ }^{5}$ indican que los conductores no respetan el paso para los peatones. Esta situación se agrava si la cantidad de autos y de peatones es elevada. Sobretodo en las horas de mayor flujo peatonal y vehicular. Esto genera gran congestionamiento, que afecta tanto al peatón como al conductor. En Lima por ejemplo, en la Avenida Javier Prado, durante las horas pico (de 7 a $9 \mathrm{~h}$ y de 15 a $19 \mathrm{~h}$ ) cruzan en ambos sentidos, unos 10000 vehículos por hora a una velocidad de $3 \mathrm{~km} / \mathrm{h}$, cuando lo normal sería $45 \mathrm{~km} / \mathrm{h}$. El flujo de peatones es de 6000 peatones por hora. ${ }^{6}$ Estas circunstancias de tráfico en las horas punta terminan estresando a los conductores que tienen que realizar paradas constantemente, con la pérdida de tiempo que ello implica, y la carga mental que se asocia a la espera, el ruido y la premura por llegar a tiempo a los lugares de destino. Los peatones por su parte, al observar este escenario, se confían y cruzan las vías por los lugares menos indicados. En este caso, es fácil darse cuenta que tanto el conductor como el peatón coadyuvan desde la posición en que se encuentra cada uno de ellos, a la ocurrencia de accidentes.

Sin embargo, es obvio que el peatón lleva las de perder, puesto que dada la colisión entre un vehículo y un peatón, este último resulta con lesiones que afectan su integridad y su salud, hasta el punto de perder la vida. Considérese que un transeúnte tiene $90 \%$ de probabilidades de sobrevivir a un accidente con un auto que lo arrolle a una velocidad de apenas $30 \mathrm{~km} / \mathrm{h}$. A velocidades superiores, las probabilidades de supervivencia decrecen hasta el $50 \%$.

Ahora bien, lo cierto es que los peatones al igual que los conductores son imprudentes y mantienen conductas de riesgo como caminar fuera de las calzadas, subir y bajar de los vehículos de transporte público en lugares no autorizados, cruzar la calle por cualquier lugar menos por las líneas peatonales y no hacer uso de los puentes peatonales de manera pertinente. Todas estas conductas ponen indudablemente en riesgo la vida y la integridad física de las personas. Pero también es cierto que estas conductas no se manifiestan de la nada, sino que responden a ciertas actitudes que a su vez se encuentran mediadas por los patrones culturales que rigen nuestras normas de convivencia.

En ese sentido, una actitud es una predisposición para responder de manera predeterminada a los estímulos relevantes. ${ }^{7}$ Las actitudes se caracterizan además porque: 1 . Son aprendidas, 2 . Son relativamente estables, 3. Implican relaciones entre sujetos o sujeto-objeto, y 4 .Tienen propiedades motivadoras-afectivas. De ahí que las actitudes motivan ciertas conductas. Pero una actitud no siempre se corresponde con la conducta. Una persona puede tener una actitud hacia "algo" pero actúa de manera opuesta en determinadas circunstancias. Por ejemplo, podemos tener una actitud negativa hacia el alcohol, pero sin embargo bebemos. En estos casos, existe una incongruencia que refleja, como diría G. Allport, ${ }^{8}$ cierto grado de inmadurez e inestabilidad, en la configuración de la personalidad.

Es importante considerar que la conducta es una manifestación externa, en tanto que las actitudes son estados psíquicos internos. Por esta razón, las actitudes son valoradas a través de autorreportes, que frecuentemente se basan en la aplicación de escalas tipo Likert, ${ }^{9}$ mientras la conducta se estudia a través del método de 
observación sistemática. ${ }^{10}$ Precisamente, se ha utilizado la observación naturalista para estudiar la conducta de las personas en los cruceros peatonales. ${ }^{11}$

En este estudio se pretende, a través de la aplicación de una encuesta, determinar las causas por las cuáles las personas no utilizan los puentes peatonales, y describir diversas variables sociodemográficas como el género, la edad y el grado de instrucción, en función de los motivos que tienen los peatones para no cruzar las vías por los puentes. Para su realización, hemos seguido el modelo del estudio de Hidalgo y otros. ${ }^{12}$

Este trabajo se ubica dentro del marco de la psicología del tránsito, la salud pública y la psicología de la seguridad.

\section{MÉTODOS}

\section{Muestra}

El presente estudio es de corte descriptivo. La muestra quedó conformada por 360 personas que transitan por las vías aledañas a tres puentes de la ciudad: 1. El puente de la Avenida Venezuela a la altura del área de sociales de la Universidad Nacional de San Agustín, 2. El puente de la Avenida Alcides Carrión frente al Hospital Regional Honorio Delgado, y 3. El puente de la Avenida Ejército a la altura del Mall Real Plaza. Se realizó un muestreo no probabilístico mediante la técnica de muestro por cuotas siguiendo las pautas de Hernández y otros. ${ }^{13}$ que plantean que para el estudio de conductas en poblaciones regionales un número aceptable es de 300 a 700 unidades de análisis.

\section{Instrumentos}

Para recoger la información, se aplicó una encuesta diseñada para los fines del estudio que recoge datos como edad, género, grado de instrucción, lugar de procedencia y una relación de alternativas sobre los motivos por los cuáles no se utilizan los puentes peatonales, entre ellos:

- Me canso mucho.

- El tiempo no me lo permite, estoy sobre la hora.

- Tengo temor de que me asalten.

- No hay necesidad, es igual cruzar por la calle.

Asimismo, la ficha de llenado de la información cuenta con un espacio para registrar cualquier otra explicación que no conste entre las alternativas de respuesta. Las respuestas además, eran excluyentes, no se podían marcar dos o más alternativas, y se incluyó una pregunta para registrar la incidencia de accidentes que ha tenido la persona encuestada. 


\section{Procedimientos}

La recolección de la información se efectúo durante los días de semana desde las 12 hasta las $14 \mathrm{~h}$. Se encuestó solo a las personas que no usaban el puente, luego de haber cruzado la pista. Para recoger la información, se contó con la participación de cinco estudiantes universitarios que fungieron como colaboradores, quienes recibieron adiestramiento para realizar esta tarea. Las encuestas se llenaron por los colaboradores, quienes explicaban y leían en todo momento su contenido a los transeúntes. Ellos se encargaban también de registrar los datos de las personas encuestadas. Solo se consideraron las encuestas de las personas que accedieron a participar emitiendo su opinión y facilitando sus datos. Se descartaron los peatones que no eran mayores de edad. Toda la información se recolectó entre octubre y noviembre del 2009.

\section{RESULTADOS}

Con respecto a los datos demográficos, se encuestaron 270 hombres (75\%) y 90 mujeres $(25 \%)$ con una edad promedio de 31,97 años $(30,15$ años para los hombres y 33,78 años para las mujeres).

Un mayor porcentaje de hombres tenía estudios secundarios y de nivel superior, en comparación con las mujeres. Además, mientras todos los hombres presentaron un grado de instrucción por encima del nivel básico de educación primaria, el 2,5\% de las mujeres solo contaba con estudios primarios. Esto supone que la escolaridad o el grado de instrucción no es un factor que garantice la prevención en materia de seguridad vial, a través de la manifestación de conductas seguras como es el uso de puentes peatonales.

Con respecto al lugar de procedencia, la mayoría de personas era de Arequipa, mientras que un bajo porcentaje procedían de Cuzco, Puno, Lima y Tacna. Menos del $3 \%$ vinieron de Moquegua, Ilo y Cajamarca. Esto quiere decir que la mayoría de peatones infractores que se encuestaron eran arequipeños, pero esto no quiere decir que la procedencia sea un factor determinante, puesto que lo ideal sería comparar nuestros datos con los que provienen de los diversos departamentos del país.

Sobre los motivos por los que los encuestados cruzaron la calle en lugar de usar el puente peatonal, independientemente del género, la respuesta más frecuente fue la falta de tiempo seguida de la pereza. Si embargo, se observaron diferencias entre hombres y mujeres en las siguientes alternativas. Para el caso de los hombres, el $20,84 \%$ indicó que no era necesario usar el puente, en tanto que el $0,83 \%$ de las mujeres marcaron esta respuesta. En cambio, el 2,5\% de mujeres señalaron que tenían temor de ser asaltadas, mientras que 18,33\% de hombres consideraron esta alternativa entre sus respuestas (tabla 1 ). Al respecto cabe señalar que las mujeres hicieron algunas observaciones al diseño del puente que son importantes, y que detallaremos más adelante. 
Tabla 1. Motivos para no usar los puentes

\begin{tabular}{|c|c|c|c|c|c|c|}
\hline \multirow{2}{*}{ Motivo } & \multicolumn{2}{|c|}{ Hombres } & \multicolumn{2}{|c|}{ Mujeres } & \multicolumn{2}{|c|}{ Total } \\
\hline & No. & $\%$ & No. & $\%$ & No. & $\%$ \\
\hline Pereza & 63 & 17,5 & 33 & 9,17 & 99 & 26,67 \\
\hline Falta de tiempo & 66 & 18,33 & 45 & 12,5 & 111 & 30,83 \\
\hline Temor de robo & 66 & 18,33 & 9 & 2,5 & 75 & 20,83 \\
\hline No es necesario & 75 & 20,84 & 3 & 0,83 & 78 & 21,67 \\
\hline Total & 270 & 75 & 90 & 25 & 360 & 100 \\
\hline
\end{tabular}

Estos datos sugieren que aunque la falta de tiempo es un factor que se debe considerar, la cuestión de los valores y la información se torna relevante, puesto que la impuntualidad y la falta de conciencia del uso de puentes peatonales, así como del cumplimiento del reglamento de tránsito, constituyen el trasfondo de las respuestas que dieron los encuestados. En ese sentido, las mujeres parecen tener mayor conciencia de seguridad vial, ya que muy pocas marcaron que no era necesario usar el puente.

El 10,83 \% de los hombres tuvieron algún tipo de accidente de tránsito previo frente al 3,33\% de mujeres que también lo tuvieron. A pesar de ello, la mayoría $(64,17 \%$ de hombres y 21,67 de mujeres), no sufrieron accidentes de tránsito previos a la encuesta.

Estos resultados globales tendrían un matiz más preciso si se considera la ubicación del puente. En tal sentido, presentamos, a continuación los resultados en función de la ubicación de los puentes peatonales.

\section{Puente peatonal de la Avenida Venezuela}

En el puente peatonal de la Avenida Venezuela, el número de personas encuestadas fue de 123 con una media de edad de 34,27 años, de las cuales $99(80,49 \%)$ eran hombres y $24(19,51 \%)$ mujeres. La media de edad para los hombres era de 31,66 años y para las mujeres 36,88 . De ello se desprende que fueron los hombres quienes usaron menos el puente de la Avenida Venezuela en comparación con las mujeres.

De acuerdo con el grado de instrucción, los transeúntes de la Avenida Venezuela poseían en su mayoría estudios superiores, tanto los hombres $(53,66 \%)$ como las mujeres $(9,75 \%)$. En menor medida se encontraban aquellos que tenían estudios secundarios $(24,16 \%$ de los hombres y $4,88 \%$ de las mujeres) y menos todavía, los que poseían solamente estudios primarios, en este último caso, fueron las mujeres quienes conformaron parte de este grupo.

En cuanto al lugar de procedencia, el $63,42 \%$ de encuestados en el puente peatonal de la Avenida Venezuela procedían de Arequipa, el $12,20 \%$ de Puno, el $9,75 \%$ de Lima, al igual que los que proceden de Tacna y el 4,88 \% venían de Cuzco. 
Los motivos por los que los peatones del área de sociales de la Universidad Nacional de San Agustín de la Avenida Venezuela no utilizaron el puente, fueron para los hombres, el temor de ser asaltados en el 24,39\%, la ausencia de necesidad en el $24,39 \%$, la falta de tiempo en el $19,61 \%$ y la pereza en el 12,20 $\%$. Para el caso de las mujeres, el motivo más frecuente fue la falta de tiempo en el $9,75 \%$, seguido del temor a ser asaltadas en el 4,88 \% y la pereza o la falta de necesidad en el $2,44 \%$. Estos datos son consistentes con los constantes robos que se producen en la zona a los estudiantes de la Universidad, precisamente es en el puente peatonal, donde los estudiantes son acosados al no tener alternativa de escape desde esta estructura. En la ubicación de este puente, la criminalidad de la zona genera desconfianza en los peatones, principalmente hombres, que optan por cruzar la pista. No debe dejarse de lado empero, que la falta de tiempo así como la percepción de que no hay necesidad de usar el puente, tienen valores porcentuales importantes.

El $87,81 \%$ de las personas encuestadas no tuvieron accidente de tránsito previo, solo la minoría de hombres como de mujeres sufrieron algún incidente de este tipo.

\section{Puente peatonal de la Avenida Alcides Carrión}

En el puente peatonal de la Avenida Alcides Carrión, ubicado al frente del Hospital Regional Honorio Delgado, se encuestaron 132 personas cuyo promedio de edad era de 29,83 años, de ellos 84 eran hombres $(63,64 \%)$ con una edad media de 27,9 años y $48(36,36 \%)$ mujeres con una edad promedio de 31,96 años.

Según el grado de instrucción, el 72,73 \% tenía estudios superiores, el $25 \%$ tenía estudios secundarios y el 2,27, estudios primarios. Los hombres alcanzaron mayor grado de instrucción en comparación con las mujeres, en tanto que el 2,27 \% de las mujeres contaba con grado de instrucción primario y ningún varón se encontraba en este nivel de escolaridad.

Por lugar de procedencia, el 68,18\% eran naturales de Arequipa, el 15,92\% de Cuzco, el 6,82 de Puno y el 9,08\% provenía de Tacna, Moquegua, Piura e Ilo.

Los motivos más frecuentes por los cuáles los peatones no utilizaban el puente de la Avenida Alcides Carrión, fueron para los hombres, la falta de tiempo (25\%), la pereza de subir y bajar del puente $(20,45 \%)$, la percepción de que no era necesario $(11,7 \%)$ y el temor de ser asaltado $(6,82 \%)$. Para las mujeres, los motivos, en orden de frecuencia fueron la falta de tiempo $(20,45 \%)$, la pereza $(13,64 \%)$ y el temor de ser asaltadas $(2,27 \%)$. Ninguna mujer respondió que no era necesario subir al puente.

Cabe mencionar que en esta locación, las mujeres encuestadas indicaron que la estructura del puente no favorecía su uso, debido a que les generaba diversos inconvenientes. En las observaciones se anotaron comentarios tales como: "no puedo subir porque visto falda y me pueden ver los hombres que están abajo" o "las ranuras de los peldaños me hacen atracar los tacos". En este caso, la ergonomía jugó un papel importante -al menos para las mujeres- como un factor que favoreció cruzar la pista en lugar de utilizar el puente peatonal.

La mayoría de las personas que conformaron la muestra $(54,55 \%$ de hombres y $31,82 \%$ de mujeres) no tuvieron accidentes de tránsito previos. 


\section{Puente peatonal de la Avenida Ejército}

Las personas entrevistadas alrededor del puente de la Avenida Ejército sumaron 105 , de ellos $87(82,86 \%)$ eran hombres y $18(17,14 \%)$ mujeres. La media de edad era de 31,08 años, 31,10 para los hombres y 32, para las mujeres.

Ninguno de los encuestados tenía de nivel primario de instrucción. El $20 \%$ de los hombres tenía nivel secundario al igual que el 2,86 \% de las mujeres. El 62,86\% de los hombres y el $14,28 \%$ de las mujeres tenía estudios superiores. Una suposición que se desprende de estos valores es que, dada la ubicación del puente, en una zona donde viven personas de mayor nivel socioeconómico, el nivel educativo es más alto, pero a pesar de ello, los hombres, quienes presentaban mayor grado de instrucción, no utilizaban el puente peatonal en mayor proporción que las mujeres.

De acuerdo al lugar de procedencia, el 68,57 \% provenían de Arequipa, en menor medida venían de Puno $(8,57 \%)$, Cuzco $(8,57 \%)$, Lima $(5,72 \%)$, Piura $(5,72 \%)$ y Cajamarca $(2,85 \%)$ para un total de $31,43 \%$.

Los motivos por los cuales los peatones no usaron el puente peatonal de la Avenida Ejército, se tiene que el $28,58 \%$ de los hombres señaló que no era necesario, el 25, $71 \%$ tenía temor de que le robaran, el $20 \%$ sentía pereza y el 8,57 \% alegó falta de tiempo. Para el caso de las mujeres, el $11,42 \%$ sentía pereza y el 5,72 \% dijo que no tenía tiempo. Ninguna mujer marcó las alternativas de temor a ser asaltada o de considerar innecesario cruzar la pista por el puente.

Por otro lado, el 17,14 \% de transeúntes han sufrido accidentes de tránsito previos en comparación con el $82,86 \%$ de las personas que no han pasado por esta experiencia. Los hombres al igual que en los casos anteriores han tenido mayor incidencia de accidentes de tránsito (tabla 2).

Tabla 2. Experiencia previa de accidentes de tránsito, Av. Ejército

\begin{tabular}{|c|c|c|c|c|c|c|}
\hline \multirow{2}{*}{ Incidencia de accidentes } & \multicolumn{2}{|c|}{ Hombres } & \multicolumn{2}{|c|}{ Mujeres } & \multicolumn{2}{|c|}{ Total } \\
\hline & No. & $\%$ & No. & $\%$ & No. & $\%$ \\
\hline Accidentes previos & 15 & 14,28 & 3 & 28,6 & 18 & 17,14 \\
\hline Sin accidentes previos & 72 & 68,58 & 15 & 14,28 & 87 & 82,86 \\
\hline Total & 87 & 82,86 & 18 & 17,14 & 105 & 100 \\
\hline
\end{tabular}

Al comparar los resultados de los motivos por los cuales los peatones no utilizaron los puentes, en función del género y su ubicación, se aprecia en la figura que las mujeres, en un mayor porcentaje, 36,6 \%, no utilizaron los puentes peatonales por pereza, en comparación con los hombres, 23,3\%.

La falta de tiempo fue también un motivo que mayoritariamente marcaron las mujeres (50 \%) en comparación con los hombres (24,4\%) (Fig.). 


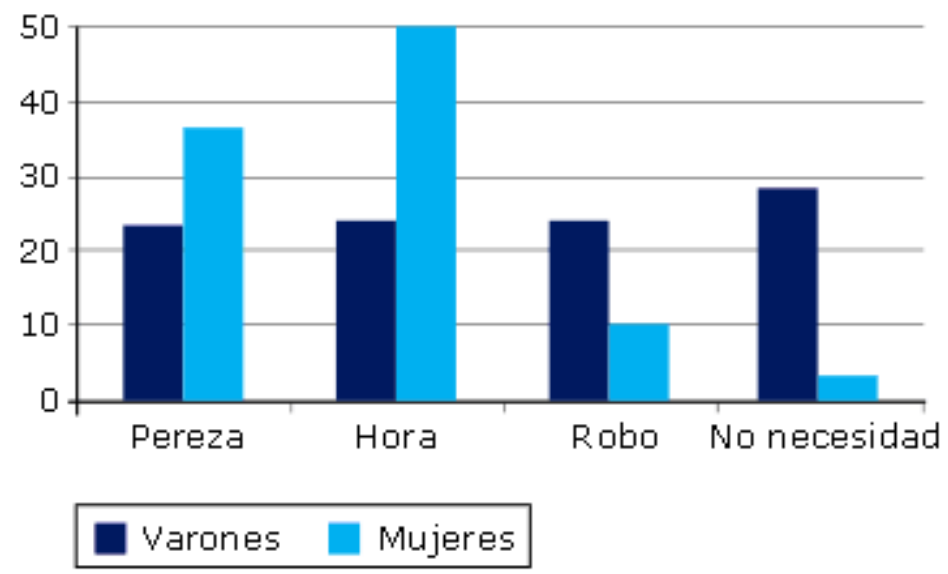

Fig. Porcentajes de los motivos de desuso de puentes según el género.

Los hombres, por otro lado, escogieron con mayor frecuencia el temor a ser asaltados $(24,4 \%)$, como motivo para no usar los puentes peatonales en comparación con las mujeres $(10 \%)$. Del mismo modo, los hombres también eligieron mayoritariamente $(27,9 \%)$ la falta de necesidad de utilizar el puente para cruzar la pista en comparación con las mujeres $(3,4 \%)$.

Estos valores, vistos ya en su conjunto sugieren que los hombres utilizaron los puentes en menor medida que las mujeres y que los motivos que explicaron estas conductas de riesgo se deben al temor de ser asaltados y a la percepción de que no era necesario usarlos para cruzar la calle. La falta de tiempo, es otro factor relevante que debe considerarse sobre todo en el caso de las mujeres, al igual que la pereza.

\section{DISCUSIÓN}

En el presente estudio, se planteó la necesidad - dentro del marco de la seguridad vial-, de conocer las causas por las que los transeúntes de Arequipa no utilizan los puentes peatonales. Se escogieron tres puentes peatonales ubicados en diferentes zonas de la ciudad y se encuestaron a los peatones, 260 hombres y 90 mujeres, que no lo utilizaron para cruzar la pista. En primer lugar, es incuestionable por el número de encuestados, que la mayoría de personas que no usan el puente son hombres. El nivel educativo no parece ser un factor determinante puesto que la mayoría de los infractores tenía estudios superiores. Ello supone entonces, que la educación debe poner mayor énfasis en la formación de personas con valores cívico-viales. Cabe mencionar que otros estudios, han publicado que las personas con grados de estudios superiores tienen mayor riesgo de sufrir accidentes y de tener heridas de mayor gravedad. ${ }^{14}$

Esto se corrobora, cuando vemos que los motivos marcados por los peatones que no utilizaron el puente, fueron principalmente la falta de tiempo $(30,83 \%)$ y el cansancio que genera el subir y bajar el puente (26,67\%). En ambos casos están presentes dos antivalores. En el primero, la falta de tiempo supone impuntualidad, mientras que el deseo de evitar el cansancio en el segundo, supone pereza. Por 
tanto, las causas de la ocurrencia de las conductas de riesgo al cruzar indebidamente las pistas, se encuentran mediadas por la falta de valores.

Existen asimismo, diferencias de género que remarcan aún más el énfasis puesto en los valores, al menos de parte de los hombres, ya que si bien es cierto, las mujeres no usan el puente mayoritariamente porque no disponen de tiempo o porque les da pereza; los hombres también aducen que no es necesario usar los puentes en el $27,9 \%$, lo cual revela de manera más preocupante la falta de conciencia en el género masculino. Aunque esto quizás se deba a un exceso de confianza, dado que la norma que regula la conducta de los peatones está vigente, el no cumplirla constituye una infracción grave, en la que subyace también un problema de valores.

Por otro lado, el temor a ser asaltado explica en el $24,4 \%$ de los hombres, el uso indebido de los puentes peatonales, en particular, del que se ubica en la Avenida Venezuela. En ese sentido, la ubicación del puente es fundamental para explicar la conducta de riesgo que conlleva a la alta accidentabilidad de los peatones. Reséndiz, ${ }^{15}$ encuentra, en base al análisis de georreferenciación, que los puentes no son utilizados debido a la posibilidad de cruzar por la calle y la inseguridad que ofrecen. Asimismo, mediante esta técnica pudo determinar que en la ciudad de México los ejes viales abarcan el 33,7 \% de los puentes peatonales, las vías anulares abarcan el $28,7 \%$ y las vías radiales el $13 \%$. Además, en los ejes viales se concentra el 33,2 \% de atropellados, en las vías locales el 29,6 y en las principales el 27,3. Es decir, que aunque resulte paradójico, existe mayor cantidad de atropellados en las vías donde se erigen los puentes peatonales.

Esto es así porque las personas no usan los puentes para cruzar la pista y los conductores por su parte se confían por la presencia del puente. La consecuencia es que tanto uno como el otro contribuyen con el aumento de la incidencia de los accidentes de tránsito. Ante este tipo de hallazgos, es fundamental tomar medidas correctivas, sino, las consecuencias son realmente trágicas. En México, según los cálculos efectuados en 1973 murieron alrededor de 8000 personas en el mismo lugar, lo cual significa que casi una persona murió por hora, día y noche, todos los días durante ese año. ${ }^{16}$ Este ejemplo ilustra cómo si no se corrigen los factores que inciden en la accidentabilidad vial, una vez detectados, los accidentes se repetirán una y otra vez hasta convertirse en cifras de estadísticas increíbles como las que hemos mostrado.

Ante este problema, se han tomado diversas medidas en los países desarrollados para propiciar el uso de los puentes peatonales. Una medida ha consistido en colocar dispensadores de caramelos en la mitad de los puentes con el fin de atraer a los peatones. Este tipo de medida está inspirada en teorías conductistas del aprendizaje, más específicamente, en las teorías del condicionamiento operante, donde la presentación de estímulos placenteros (caramelos) después de una conducta, refuerzan su ocurrencia. Otras medidas han comprendido la colocación de barreras, para evitar que los peatones crucen por la pista. En ese sentido, las modificaciones del ambiente inseguro, como la colocación de barreras se asocian al descenso de la mortalidad por atropellamiento hasta en el $10 \%$. De hecho los puentes con mayor índice de accidentes carecen de barreras. ${ }^{17}$

En ese sentido, en el puente de la Avenida Venezuela, aunque existen barreras, no cubren un tramo largo, de modo que los peatones prefieren circundar las bardas y cruzar por la calle. Una medida importante sería extender las barreras de seguridad por unos cuantos metros más, para que frente a esta dificultad, el uso del puente resulte menos fatigoso y obviamente también más seguro, en comparación con rodear las bardas. De hecho, actualmente, las barreras de seguridad se han 
extendido en esta zona y se puede apreciar que el flujo de personas que utilizan el puente peatonal ha aumentado en tanto que ha disminuido el número de personas que cruzan por la pista.

Además sería conveniente colocar seguridad permanente en las inmediaciones de este puente, puesto que la mayoría de peatones hombres indicó que no lo utilizaban porque tenían temor de ser asaltados. Es evidente que cada puente en el que se realizaron las encuestas presenta un complejo multifactorial que muestra un muy particular panorama respecto de las causas por las cuales los peatones no utilizan los puentes. Así por ejemplo, en el puente de la Avenida Alcides Carrión se registró la mayor cantidad de mujeres que no usan el puente. Si bien los motivos suelen presentarse como en los otros casos, en función de la pereza y la falta de tiempo de los peatones, se tomó nota de diversas quejas de las mujeres que no utilizaron el puente, quienes señalaron que el diseño del mismo dificulta su uso y motiva el tránsito por la pista. En ese sentido, el factor ergonómico parece jugar un papel importante para las personas de sexo femenino que transitan por este puente y que además presentan características diferenciales, como por ejemplo ser jóvenes, movilizarse a su trabajo en bus o combi, vestir falda o minifalda y usar zapatos de taco alto.

Llama la atención que los resultados del puente ubicado en la Avenida Ejército sean muy distintos de los esperados, ya que un elevado numero de los hombres encuestados $(54,29 \%)$ dieron de manera más frecuente por respuesta que tienen temor de ser asaltados y que no era necesario usar el puente. En el caso de las mujeres ninguna persona optó por estas alternativas de respuesta a diferencia de las otras locaciones, donde, aunque en un muy bajo porcentaje, se registraron respuestas similares entre las mujeres.

Por otro lado, nuestros resultados son equiparables a los encontrados en otras investigaciones publicadas. Hidalgo y otros, ${ }^{12}$ también valoran las razones de no uso de los puentes peatonales de la ciudad de México, en función de 4 alternativas de respuesta: son inseguros, puedo cruzar la calle, flojera, no puedo hacerlo y otras razones. Encontraron que el $24,8 \%$ aduce que no usan el puente peatonal por falta de seguridad, $25,7 \%$ indica no es necesario, $30,7 \%$ no cruza el puente porque le da flojera, $13,8 \%$ dice que no puede hacerlo y $5 \%$ brindó otra explicación. Como se aprecia, los resultados son similares a los que hemos informado.

Para terminar, cabe recordar que aproximadamente el $77 \%$ de los accidentes se producen en zonas urbanas, ${ }^{17}$ y que uno de los sitios de mayor ocurrencia de accidentes son los puentes peatonales. ${ }^{1}$

En el presente estudio se pudo determinar algunos motivos que inciden en las conductas de riesgo de las personas que no utilizan los puentes peatonales, como son la falta de tiempo, la pereza, el temor a ser asaltados o la falta de necesidad. Con la información obtenida se deben tomar medidas que abarcan la seguridad (colocación de bardas de protección en las bermas de las avenidas principales) y vigilancia (ubicación de efectivos policiales). Es también necesario, implementar campañas de concientización que brinden información a los peatones sobre los riesgos a que se enfrentan por cruzar indebidamente la calle, y por infringir la ley. 


\section{REFERENCI AS BI BLI OGRÁFICAS}

1. Alfaro D. Problemática sanitaria y social de la accidentalidad del transporte terrestre. Rev Peru Med Exp Salud Publica. 2008;25(1):133-7.

2. González D. Medicina y accidentes de tránsito. Rev Med Hered. 2003; 14(2):51-2.

3. Arias WL. Una reseña introductoria a la psicología del tránsito. Rev Psicol (Trujillo). 2011; 13. De próxima aparición.

4. Rivas G. Realidades y urgencias del transporte público en Arequipa. Punto de Vista. 2010;2(6):7-8.

5. Conejera M, Donoso D, Moyano E, Peña J, Saavedra F. Comunicación persuasiva y cambio de actitudes hacia la seguridad de tránsito en peatones. Rev Latin Psicol. 2003; 35(1): 77-90.

6. Santos de la Cruz E. Contaminación sonora por ruido vehicular en la Avenida Javier Prado. Ind data. 2007;10(1):11-5.

7. Whittaker JO. Psicología. 2da ed. México, D.F.: Nueva Editorial Interamericana; 1971.

8. Allport GW. La personalidad, su configuración y desarrollo. Barcelona: Herder; 1973.

9. Méndez LM, Peña JA. Manual práctico para el diseño de la Escala de Likert. México, D.F.: Trillas; 2006.

10. Arnau J. Psicología experimental. Un enfoque metodológico. México D.F.: Trillas; 1982.

11. Schultz DP. Psicología industrial. Colombia: McGraw-Hill; 1998.

12. Hidalgo E, Campuzano J, Rodríguez JM, Chias L, Reséndiz $H$, Sánchez $H$, et al. Motivos de uso y no uso de puentes peatonales en la Ciudad de México: La perspectiva de los peatones. Salud Pública Mex. 2010;52(6):502-10.

13. Hernández R, Fernández C, Baptista P. Metodología de la investigación. México, D.F.: McGraw-Hill; 1997.

14. Hidalgo E, Híjar M, Blanco J, Kageyama ML. Factores asociados con la gravedad de lesiones ocurridas en la vía pública en Cuernavaca, Morelos, México. Salud Pública Mex. 2005; 47(1):30-8.

15. Reséndiz HD. Georreferenciación de puentes peatonales en ciudad de México y su relación con peatones atropellados [Internet]. México; 2003 [citado Abr 2011]. Disponible en: http://www.institutoivia.com/cisevponencias/analisis_accidentes_aa/Hector_Resendiz.pdf 
16. Alfaro C, Díaz C. Los accidentes de tránsito: Creciente problema para la salud pública. Rev Panam Salud Pública. 1977;83(4):310-8.

17. Híjar MC. Utilidad del análisis geográfico en el estudio de las muertes por atropellamiento. Salud Pública Mex. 2000;42(3): 188-93.

Recibido: 12 de octubre de 2011.

Aprobado: 28 de octubre de 2011.

Walter L. Arias Gallegos. Universidad Católica San Pablo. Urb. Campiña Paisajista s/n Quinta Vivanco, Cercado. Arequipa, Perú.

Teléf.: (51-54) 608-020. Correos electrónicos: warias@ucsp.edu.pe,

walterlizandro@hotmail.com 